\title{
Hall's criterion for nilpotence in semi-abelian categories
}

\author{
J. R. A. Gray
}

May 7, 2019

\begin{abstract}
A well-known theorem of P. Hall, usually called Hall's criterion for nilpotence, states: a group $G$ is nilpotent whenever it has a normal subgroup $N$ such that $G /[N, N]$ and $N$ are nilpotent. We widely generalize this result, replacing groups with objects in an abstract semi-abelian category satisfying suitable conditions. In particular, these conditions are satisfied in any algebraically coherent semi-abelian category, and hence in many categories of classical algebraic structures (including a few where Hall's theorem is already known). Note that the categories of crossed modules, crossed Lie algebras, $n$-cat groups, and cocommutative Hopf algebras over fields are also algebraically coherent. We give, however, a counter-example showing that Hall's theorem does not hold in the (semiabelian) variety of non-associative rings.
\end{abstract}

\section{Introduction}

A well-known theorem of P. Hall [14] (see also D. J. S. Robinson [25]), called Hall's criterion for nilpotence, states: a group $G$ is nilpotent whenever it has a normal subgroup $N$ such that $G /[N, N]$ and $N$ are nilpotent. Analogous results for Lie algebras and for certain types of non-associative algebras were proved by C. Y. Chao [5] and E. L. Stitzinger [26, respectively. Hall's theorem can be reformulated as a weak form of extension closedness for the class of nilpotent groups: if $p: E \rightarrow B$ is a surjective group homomorphism with nilpotent $B$ and the kernel contained in the commutator $[N, N]$ of a normal nilpotent subgroup $N$ of $E$, then $E$ is a nilpotent group.

This paper is devoted to a wide generalization of this fact (Theorem 3.4): we replace a surjective homomorphism with a regular epimorphism in an abstract semi-abelian category [18] satisfying (i) Huq and Higgins commutators of normal monomorphisms coincide; (ii) Huq commutators distribute over binary joins (restricted to normal monomorphisms); (iii) the Jacobi identity (Condition 3.1 (b)(ii)) holds for Huq commutators. These conditions turn out to be satisfied in any semi-abelian category that is algebraically coherent in the sense of [7], and therefore in each of the above-mentioned concrete categories as well as in 
all categories of interest in the sense of G. Orzech [23. Note that algebraically coherent semi-abelian categories include the categories of: cocommutative Hopf algebras over an arbitrary field, (pre)crossed modules, crossed Lie algebras, and $n$-cat groups in the sense of J.-L. Loday [20] - see Remark 3.2 for further details and references.

Our approach substantially uses what we call Jacobi commutator semilattices; it is a notion intermediate between commutator posets and associative commutator lattices in the sense of E. Mehdi-Nezhad [22].

\section{Jacobi commutator semi-lattices}

In this section we introduce a 'theory of Jacobi commutator semi-lattices' and develop it essentially as far as necessary for the purposes of Section 3, where we use it in the proof of our main result on nilpotent objects in a semi-abelian category.

Definition 2.1. (c.f. Definition 4.1 of [22]) $A$ commutator semi-lattice is a triple $(X, \leq, \cdot)$ where $X$ is a set, $\leq$ is a binary relation on $X$, and $\cdot$ is a binary operation on $X$ satisfying:

(a) $(X, \leq)$ is a join semi-lattice;

(b) the operation - is commutative;

(c) for each $a, b \in X, a \cdot b \leq b$;

(d) for each $a, b, c \in X, a \cdot(b \vee c)=(a \cdot b) \vee(a \cdot c)$.

Remark 2.2. Note that for a commutator semi-lattice $(X, \leq, \cdot)$ and an element $x$ in $X$, the map

$$
\begin{aligned}
& X \stackrel{x \cdot-}{\longrightarrow} X \\
& y \longmapsto x \cdot y
\end{aligned}
$$

is order preserving.

Definition 2.3. A commutator semi-lattice $(X, \leq, \cdot)$ is a Jacobi commutator semi-lattice if

(a) for each $a, b, c \in X, a \cdot(b \cdot c) \leq((a \cdot b) \cdot c) \vee(b \cdot(a \cdot c))$;

and is an associative commutator semi-lattice if

(b) for each $a, b, c \in X, a \cdot(b \cdot c)=(a \cdot b) \cdot c$.

Definition 2.4. A derivation of a commutator semi-lattice $(X, \leq, \cdot)$ is a map $f: X \rightarrow X$ which preserves joins and satisfies:

(a) for each $a, b \in X, f(a \cdot b) \leq(f(a) \cdot b) \vee(a \cdot f(b))$. 
$A$ derivation $f$ of a commutator semi-lattice $(X, \leq, \cdot)$ is an inner derivation if there exists $x$ in $X$ such that $f=x \cdot-$, that is, for each a in $X, f(a)=x \cdot a$.

Remark 2.5. Note that for a commutator semi-lattice $(X, \leq, \cdot)$ :

(a) the Jacobi identity (Condition 2.3 (国)) easily follows from associativity (Condition 2.3 (b) ), and is equivalent to the condition: for each $x$ in $X$, the map $x \cdot-$ is an inner derivation of $(X, \leq, \cdot)$

(b) a map $f: X \rightarrow X$ which is bounded above by $1_{X}$ (that is $f(x) \leq x$ for each $x$ in $X)$ and preserves joins is necessarily a derivation if for each $a, b \in X$, $f(a \cdot b) \leq f(a) \cdot f(b)$.

It is easy to see that every complete lattice admits a largest binary operation making it into a commutator lattice. On the other hand it doesn't seem in general possible to construct a largest binary operation making it into a Jacobi commutator semi-lattice. However, binary meet is the largest such operation when $(X, \leq)$ is a distributive lattice. In fact we have:

Proposition 2.6. If $(X, \leq)$ is a distributive lattice, then $(X, \leq, \wedge)$ is a associative commutator lattice and a morphism $f:(X, \leq) \rightarrow(X, \leq)$ which preserves joins is a derivation if and only if it is bounded above by $1_{X}$.

Proof. It is immediate that $(X, \leq, \wedge)$ is a associative commutator lattice. On the other hand if $f$ is a derivation, then for each $a$ in $X, f(a)=f(a \wedge a) \leq$ $f(a) \wedge a \leq a$ and so $f$ is bounded above by $1_{X}$. The converse follows from Remark 2.5 (b) since $f$, being an order preserving map, satisfies the condition: for each $a, b \in X, f(a \wedge b) \leq f(a) \wedge f(b)$.

Let us note as an aside related to commutator theory of arithmetical categories that:

Proposition 2.7. For a commutator semi-lattice $(X, \leq, \cdot)$ if $\cdot$ is idempotent, then $a \cdot b$ is the meet of $a$ and $b$, and $(X, \leq)$ is a distributive lattice.

Proof. For $w, a, b$ in $X$. If $w \leq a$ and $w \leq b$, then $w=w \cdot w \leq a \cdot b$. Conversely if $w \leq a \cdot b$, then trivially $w \leq a$ and $w \leq b$.

The remainder of this section is devoted to showing that for $f$ a derivation bounded above by $1_{X}$ and for $g=x \cdot-$ an inner derivation of a Jacobi commutator semi-lattice $(X, \leq, \cdot)$, if for some $y \geq x$ in $X$ and some positive integer $m, f^{m}(y) \leq g(x)$, then for each positive integer $k$ there exists a positive integer $m_{k}$ such that $f^{m_{k}}(y) \leq g^{k}(x)$. Our proof of this fact depends on a few facts.

By a routine argument using induction we obtain:

Proposition 2.8. Let $f$ be a derivation of a commutator semi-lattice $(X, \leq, \cdot)$. For each $a, b$ in $X$ and for each non-negative integer $n$ we have

$$
f^{n}(a \cdot b) \leq \vee_{i=0}^{n} f^{i}(a) \cdot f^{n-i}(b) .
$$


Lemma 2.9. Let $f$ be a derivation of a Jacobi commutator semi-lattice $(X, \leq, \cdot)$ bounded above by $1_{X}$, let $x$ be an element of $X$, and let $g: X \rightarrow X$ be the map defined by $g(s)=x \cdot s$. If for some positive integer $m, f^{m}(x) \leq g(x)$, then for each positive integer $k$,

$$
f^{n_{k}}\left(g^{k-1}(x)\right) \leq g^{k}(x)
$$

where $n_{k}=k(m-1)+1$.

Proof. We prove the claim by induction on $k$. Since $n_{1}=m$ the claim holds by assumption when $k$ is 1 . Suppose (1) holds for some positive integer $k$. By the previous proposition we have

$$
f^{n_{k+1}}\left(g^{k}(x)\right)=f^{n_{k+1}}\left(x \cdot g^{k-1}(x)\right) \leq \vee_{i=0}^{n_{k+1}} f^{i}(x) \cdot f^{n_{k+1}-i}\left(g^{k-1}(x)\right) .
$$

However, for $i \leq m-1$ since $n_{k+1}-i \geq n_{k}$ we have

$$
f^{n_{k+1}-i}\left(g^{k-1}(x)\right) \leq f^{n_{k}}\left(g^{k-1}(x)\right) \leq g^{k}(x)
$$

and hence $f^{i}(x) \cdot f^{n_{k+1}-i}\left(g^{k-1}(x)\right) \leq x \cdot g^{k}(x)=g^{k+1}(x)$. On other hand if $i \geq m$, then $f^{i}(x) \leq g(x)=x \cdot x$ and so

$$
\begin{aligned}
f^{i}(x) \cdot f^{n_{k+1}-i}\left(g^{k-1}(x)\right) & \leq(x \cdot x) \cdot g^{k-1}(x) \\
& =g^{k-1}(x) \cdot(x \cdot x) \\
& \leq\left(\left(g^{k-1}(x) \cdot x\right) \cdot x\right) \vee\left(x \cdot\left(g^{k-1}(x) \cdot x\right)\right) \\
& =g^{k+1}(x) .
\end{aligned}
$$

Therefore, $f^{n_{k+1}}\left(g^{k}(x)\right) \leq g^{k+1}(x)$ and the claim holds by induction.

Proposition 2.10. Let $f$ be a derivation of a Jacobi commutator semi-lattice $(X, \leq, \cdot)$ bounded above by $1_{X}$, let $x$ and $y$ be elements of $X$, and let $g$ be the inner derivation of $(X, \leq, \cdot)$ defined for each $s$ in $X$ by $g(s)=x \cdot s$. If $x \leq y$ and for some positive integer $m, f^{m}(y) \leq g(x)$, then for each positive integer $k$,

$$
f^{m_{k}}(y) \leq g^{k}(x)
$$

where $m_{k}=\frac{k(k+1)}{2}(m-1)+k$.

Proof. We prove the claim by induction on $k$. Since $m_{1}=m$ the claim holds by assumption when $k=1$. Suppose (2) holds for some positive integer $k$. Since $f^{m}(x) \leq f^{m}(y) \leq g(x)$ and $m_{k+1}=n_{k+1}+m_{k}$ where $n_{k+1}$ is as in the previous lemma it follows by the previous lemma that

$$
f^{m_{k+1}}(y)=f^{n_{k+1}}\left(f^{m_{k}}(y)\right) \leq f^{n_{k+1}}\left(g^{k}(x)\right) \leq g^{k+1}(x) .
$$

This completes the induction step and hence the proof of the claim. 


\section{The main result}

In this section we prove our main result which as mentioned uses Proposition 2.10 for its proof.

Let us recall the necessary background to explain precisely in which context we will prove our generalization. Semi-abelian categories were introduced, by G. Janelidze, L. Marki, W. Tholen in [18, to play a similar role for the categories of groups and algebras, as abelian categories play for the categories of abelian groups and modules. A category $\mathbb{C}$ is semi-abelian if it is pointed, Barr-exact [1], Bourn protomodular [2] and has finite coproducts. In a semi-abelian category there is a natural notion of when a pair of morphisms with common codomain commute. Note that this notion was first considered by S. Huq [15, 16] in a closely related context. To recall how this notion is defined let us introduce some notation. We denote by 0 a zero object in $\mathbb{C}$, that is, an object which is both terminal and initial. We also denote by 0 each zero morphism, that is, a morphism which factors through a zero object. For $A$ and $B$ in $\mathbb{C}$ we denote by $\left(A \times B, \pi_{1}, \pi_{2}\right)$ a product of $A$ and $B$ in $\mathbb{C}$, and write $\langle 1,0\rangle: A \rightarrow A \times B$ and $\langle 0,1\rangle: B \rightarrow A \times B$ for the unique morphisms with $\pi_{1}\langle 1,0\rangle=1_{A}, \pi_{2}\langle 1,0\rangle=0$, $\pi_{1}\langle 0,1\rangle=0$ and $\pi_{2}\langle 0,1\rangle=1_{B}$. A pair of morphisms $f: A \rightarrow C$ and $g: B \rightarrow C$ in $\mathbb{C}$, commute, if there is a (necessarily unique) morphism $\varphi: A \times B \rightarrow C$ making the diagram

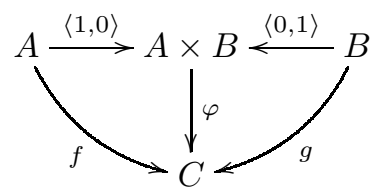

commute. More generally the Huq commutator of $f: A \rightarrow C$ and $g: B \rightarrow$ $C$ is defined to be the smallest normal subobject $N$ of $C$ such that $q f$ and $q g$ commute, where $q: C \rightarrow C / N$ is the cokernel of the associated normal monomorphism $N \rightarrow C$. It turns out that Huq commutators always exist in semi-abelian categories and, as shown by D. Bourn in [3], the commutator quotient $q: C \rightarrow C / N$ can be constructed as part of the colimiting cone of the outer arrows of (3). For subobjects $S$ and $T$ of an object $C$ in $\mathbb{C}$ we will write $[S, T]_{C}$ for the Huq commutator of the associated monomorphisms $S \rightarrow C$ and $T \rightarrow C$. We write $\operatorname{Sub}(C)$ and $\operatorname{NSub}(C)$ for the lattices of subobjects and normal subobjects of $C$, respectively. Recall also that $C$ is nilpotent [15] if there exists a non-negative integer $n$ such that $\gamma_{C}^{n}(C)=0$, where $\gamma_{C}$ is the map sending $S$ in $\operatorname{Sub}(C)$ to $[C, S]_{C}$ in $\operatorname{Sub}(C)$. The least such $n$ is the nilpotency class of $C$.

We will prove our generalization in the context of a semi-abelian category $\mathbb{C}$ satisfying the following conditions:

\section{Condition 3.1.}

(a) for $K, L \leq S \leq C$, if $K$ and $L$ are normal in $C$, then $[K, L]_{S}=[K, L]_{C}$ (in $\operatorname{Sub}(C)$ ); 
(b) for $K, L, M$ normal in $C$ :

(i) $[K, L \vee M]_{C}=[K, L]_{C} \vee[K, M]_{C}$ in $\operatorname{Sub}(C)$;

(ii) $\left[K,[L, M]_{C}\right]_{C} \leq\left[[K, L]_{C}, M\right]_{C} \vee\left[L,[K, M]_{C}\right]_{C}$ in $\mathbf{S u b}(C)$.

Before we state and prove our generalization, let us briefly recall how each of these conditions fits into the literature and make a few other comments:

\section{Remark 3.2.}

- According to Theorem 2.8 of [6], Condition 3.1 (a) is equivalent to the coincidence of the Huq and Higgins commutators of normal subobjects;

- If $\mathbb{C}$ is a semi-abelian algebraically cartesian closed category (in the sense of [4], first considered in [10, 11]), then $\mathbb{C}$ has centralizers and hence Condition 3.1 (b) (i) holds (see [12]);

- If $\mathbb{C}$ is semi-abelian and the Huq and Smith-Pedicchio commutators coincide (in the sense of [21]), then Condition [3.1 (b) (i) again holds, since the Smith-Pedicchio commutator distributes over joins in an exact Mal'tsev category with coequalizers [24].

- We call Condition 3.1 (b) (ii), the Jacobi identity. It is a special case of the so-called three subgroups lemma in the context of the category of groups. Note that according to P. Hall in [14] in the context of groups what we are calling the Jacobi identity was first proved by himself in [13], while the above mentioned three subgroups lemma was proved later by L. Kaluzhnin in [19];

- Condition 3.1 follows in a semi-abelian category from algebraic coherence in the sense of [7] (see Theorems 6.18, 6.27 and 7.1 there);

- Examples of algebraically coherent semi-abelian categories include the categories of groups, rings, Lie algebras over a commutative ring, and all categories of interest in the sense of G. Orzech [23]. The category of cocommutative Hopf algebras over an arbitrary field has been shown by M. Gran, F. Sterck and J. Vercruysse to be algebraically coherent and semi-abelian [9]. Further examples can be obtained from the fact that if $\mathbb{C}$ is an algebraically coherent semi-abelian category, then so is: each functor category $\mathbb{C}^{\mathbb{I}}$ for an arbitrary category $\mathbb{I}$; each full subcategory of $\mathbb{C}$ closed under products and subobjects; each category of split epimorphism in $\mathbb{C}$ with codomain $B$ where $B$ is an object in $\mathbb{C}$ (see Corollary 3.5 and Propositions 3.6 and 3.7 of [7]). In particular, it follows that the categories of (pre)crossed modules (of groups), crossed Lie algebras, and n-cat groups (in the sense of J.-L. Loday [20]) are all examples, as well as, each category of internal (pre)crossed modules (in the sense of G. Janelidze in [17]) of an algebraically coherent semi-abelian category.

Recalling that in a semi-abelian category the binary join of normal subobjects as subobjects is necessarily normal, we see that Condition 3.1 implies: 
Proposition 3.3. If $\mathbb{C}$ is a semi-abelian category satisfying Condition 3.1, then for each object $C$ in $\mathbb{C}$ the triple $(\operatorname{NSub}(C), \leq, \cdot)$ where $(\mathbf{N S u b}(C), \leq)$ is the lattice of normal subobjects of $C$, and $\cdot$ is defined by $K \cdot L=[K, L]_{C}$ is a Jacobi commutator semi-lattice.

As mentioned in Remark 3.2 Theorems $6.18,6.27$ and 7.1 of 7 imply that every algebraically coherent semi-abelian category satisfies Condition 3.1 We have:

Theorem 3.4. Let $\mathbb{C}$ be an algebraically coherent semi-abelian category (more generally a semi-abelian category satisfying Condition [3.1) and let $p: E \rightarrow B$ be an extension of a nilpotent object $B$ in $\mathbb{C}$. If the kernel of $p$ is contained in the Huq commutator $[N, N]_{N}$ of a nilpotent normal subobject $N$ of $E$, then $E$ is nilpotent. Furthermore, if $N$ is of nilpotency class $c$ and $B$ is of nilpotency class $d$, then $E$ is of nilpotency class at most $\frac{c(c+1)}{2}(d-1)+c$.

Proof. Let $X=\operatorname{NSub}(E)$ and let $f: X \rightarrow X$ and $g: X \rightarrow X$ be the maps defined by $f(K)=[E, K]_{E}$ and $g(K)=[N, K]_{E}$. Since Huq commutators are preserved by regular image along regular epimorphisms (see e.g. Theorem 5.2 of [8]) it follows that if $B$ is nilpotent of nilpotency class $d$, then $p\left(f^{d}(E)\right)=0$ and so $f^{d}(E)$ is a subobject of the kernel of $p$ and hence a subobject of $g(N)$. Since $(X, \leq, \cdot)$, with the corresponding interpretation as in Proposition 3.3. is a Jacobi commutator semi-lattice, and hence $f$ is a derivation bounded above by $1_{X}$, it follows from Proposition 2.10 that for any positive integer $c$,

$$
f^{\frac{c(c+1)}{2}(d-1)+c}(E) \leq g^{c}(N) .
$$

The claim is completed by noting that by Condition 3.1 (a) for $K \leq N, g(K)=$ $[N, K]_{N}$ and hence if $N$ is nilpotent of nilpotency class $c$, then $g^{c}(N)=0$.

Let us end by showing that the previous theorem does not hold in the category of non-associative rings (i.e. not necessarily associative distributive rings). As explained at the end of [12, the category of non-associative rings is a semiabelian category satisfying Condition 3.1 (b) (i) which is not algebraically cartesian closed (note the misprint there: $i_{3}$ should be $\langle 0,0,1,0\rangle$ ).

Example 3.5. Let $B$ and $E$ be the non-associative rings with underlying abelian groups the free abelian groups on $\left\{b_{1}, b_{2}\right\}$ and $\left\{e_{1}, e_{2}, e_{3}\right\}$, respectively, and with multiplication defined on generators by

\begin{tabular}{l|ll}
$\cdot$ & $b_{1}$ & $b_{2}$ \\
\hline$b_{1}$ & 0 & 0 \\
$b_{2}$ & 0 & 0
\end{tabular}

\begin{tabular}{l|lll}
$\cdot$ & $e_{1}$ & $e_{2}$ & $e_{3}$ \\
\hline$e_{1}$ & 0 & 0 & 0 \\
$e_{2}$ & 0 & $e_{3}$ & 0 \\
$e_{3}$ & $e_{3}$ & 0 & 0.
\end{tabular}

Note that this makes $B$ abelian and hence nilpotent. An easy calculation shows that unique abelian group homomorphism $p: E \rightarrow B$ with $p\left(e_{1}\right)=b_{1}, p\left(e_{2}\right)=b_{2}$ and $p\left(e_{3}\right)=0$ is a non-associative ring homomorphism. We will show that $p$ 
satisfies the remaining requirements of the theorem but $E$ is not nilpotent. It is easy to see that the kernel of $p$ is $X=\left\langle\left\{e_{3}\right\}\right\rangle$ (i.e. the subgroup of $E$ generated by $\left.e_{3}\right)$. Now let $N=\left\langle\left\{e_{2}, e_{3}\right\}\right\rangle$ and note that it is a subring of $E$. Recall that if $S$ and $T$ are subrings of a non-associative ring $R$, then the Huq commutator $[S, T]_{R}$ is the smallest ideal of $R$ containing $\{s \cdot t \mid s \in S, t \in T\} \cup\{t \cdot s \mid s \in S, t \in T\}$. Since $e_{2}^{2}=e_{3}$ and $e_{3} e_{1}=e_{3}$ it follows that $X$ is contained in both $[N, N]_{N}$ and $[E, X]_{X}$. On the other hand since $\left\{e_{i} \cdot e_{j} \mid i, j \in\{1,2,3\}\right\}=\left\{0, e_{3}\right\}$ it follows that $[N, N]_{N}=[E, E]_{E}=[E, X]_{E}=X$. Since $e_{3}^{2}=e_{3} \cdot e_{2}=e_{2} \cdot e_{3}=0$ it follows that $[N, X]_{E}=0$. Therefore $\left[N,[N, N]_{N}\right]_{N}=[N, X]_{N}=0$ and $\left[E,[E, E]_{E}\right]_{E}=[E, X]_{E}=X=[E, E]_{E}$ and so $N$ nilpotent but $E$ is not.

\section{References}

[1] M. Barr, Exact categories, in: Lecture Notes in Mathematics 236, 1-120, 1971.

[2] D. Bourn, Normalization equivalence, kernel equivalence and affine categories, Springer Lecture Notes Math. 1488, 43-62, 1991.

[3] D. Bourn, Commutator theory in strongly protomodular categories, Theory and Applications of Categories 13(2), 24-40, 2004.

[4] D. Bourn and J. R. A. Gray, Aspects of algebraic exponentiation, Bulletin of the Belgian Mathematical Society 19(5), 821-844, 2012.

[5] C.-Y. Chao, Some characterizations of nilpotent Lie algebras, Mathematische Zeitschrift 103, 40-42, 1968.

[6] A. S. Cigoli, J. R. A. Gray, and T. Van der Linden, On the normality of Higgins commutators, Journal of Pure and Applied Algebra 219(4), 897912, 2015.

[7] A. S. Cigoli, J. R. A. Gray, and T. Van der Linden, Algebraically coherent categories, Theory and Applications of Categories 30(54), 1864-1905, 2015.

[8] M. Gran, G. Janelidze, and A. Ursini, Weighted commutators in semiabelian categories, Journal of Algebra 397(Supplement C), 643-665, 2014.

[9] M. Gran, F. Sterck, and J. Vercruysse, A semi-abelian extension of a theorem by takeuchi, Journal of Pure and Applied Algebra, Online First, 2019.

[10] J. R. A. Gray, Algebraic exponentiation and internal homology in general categories, Ph.D. thesis, University of Cape Town, 2010.

[11] J. R. A. Gray, Algebraic exponentiation in general categories, Applied Categorical Structures 20(6), 543-567, 2012.

[12] J. R. A. Gray, A note on the distributivity of the Huq commutator over finite joins, Applied Categorical Structures 22(2), 305-310, 2014. 
[13] P. Hall, A contribution to the theory of groups of prime-power order, Proceedings of the London Mathematical Society 36(2), 29-95, 1933.

[14] P. Hall, Some sufficient conditions for a group to be nilpotent, Illinois Journal of Mathematics 2(4B), 787-801, 1958.

[15] S. A. Huq, Commutator, nilpotency and solvability in categories, Quarterly Journal of Mathematics 19(1), 363-389, 1968.

[16] S. A. Huq, Upper central series in a category, Journal für die reine und angewandte Mathematik 252, 209-214, 1971.

[17] G. Janelidze, Internal crossed modules, Georgian Mathematical Journal 10(1), 99-114, 2003.

[18] G. Janelidze, L. Márki, and W. Tholen, Semi-abelian categories, Journal of Pure and Applied Algebra 168, 367-386, 2002.

[19] L. Kaloujnine, Sur quelques propriétés des groupes d'automorphismes d'un groupe abstrait. (Généralisation d'un theorème de M. Ph. Hall), Comptes Rendus de l'Acadmie des Sciences Paris 231, 400-402, 1950.

[20] J.-L. Loday, Spaces with finitely many non-trivial homotopy groups, Journal of Pure and Applied Algebra 24(2), 179 - 202, 1982.

[21] N. Martins-Ferreira and T. Van der Linden, A note on the "Smith is Huq" condition, Applied Categorical Structures 20(2), 175-187, 2012.

[22] E. Mehdi-Nezhad, Abstract annihilation graphs, Cahiers de Topologie et Géométrie Différentielles Catégoriques 56, 133-146, 2015.

[23] G. Orzech, Obstruction theory in algebraic categories I, II, Journal of Pure and Applied Algebra 2(4), 287-314 and 315-340, 1972.

[24] M. C. Pedicchio, A categorical approach to commutator theory, Journal of Algebra 177(3), 143-147, 1995.

[25] D. J. S. Robinson, A property of the lower central series of a group, Mathematische Zeitschrift 107, 225-231, 1968.

[26] E. L. Stitzinger, On nilpotent algebras, Illinois J. Math. 22(3), 499-505, 1978. 